\title{
Spectral properties of some regular boundary value problems for fourth order differential operators
}

Research Article

Nazim B. Kerimov ${ }^{1 *}$, Ufuk Kaya ${ }^{1+}$

1 Department of Mathematics, Faculty of Science and Letters, Mersin University, Mersin, 33343, Turkey

Received 3 August 2011 ; accepted 30 January 2012

Abstract: In this paper we consider the problem

$$
\begin{aligned}
& y^{\mathrm{IV}}+p_{2}(x) y^{\prime \prime}+p_{1}(x) y^{\prime}+p_{0}(x) y=\lambda y, \quad 0<x<1, \\
& y^{(s)}(1)-(-1)^{\sigma} y^{(s)}(0)+\sum_{l=0}^{s-1} \alpha_{s, l} y^{(l)}(0)=0, \quad s=1,2,3, \\
& y(1)-(-1)^{\sigma} y(0)=0,
\end{aligned}
$$

where $\lambda$ is a spectral parameter; $p_{j}(x) \in L_{1}(0,1), j=0,1,2$, are complex-valued functions; $\alpha_{s, l}, s=1,2,3$, $l=\overline{0, s-1}$, are arbitrary complex constants; and $\sigma=0,1$. The boundary conditions of this problem are regular, but not strongly regular. Asymptotic formulae for eigenvalues and eigenfunctions of the considered boundary value problem are established in the case $\alpha_{3,2}+\alpha_{1,0} \neq \alpha_{2,1}$. It is proved that the system of root functions of this spectral problem forms a basis in the space $L_{p}(0,1), 1<p<\infty$, when $\alpha_{3,2}+\alpha_{1,0} \neq \alpha_{2,1}, p_{j}(x) \in W_{1}^{j}(0,1), j=1,2$, and $p_{0}(x) \in L_{1}(0,1)$; moreover, this basis is unconditional for $p=2$.

MSC: $\quad 34 B 05,34 L 20,34 L 10$

Keywords: Fourth order eigenvalue problem $•$ Not strongly regular boundary conditions $•$ Asymptotic behavior of eigenvalues and eigenfunctions - Basis properties of the system of root functions

(C) Versita Sp. z o.o.

\section{Introduction}

It is known $[7,14,26]$ that the system of root functions of an arbitrary even order differential operator with strongly regular boundary conditions forms an unconditional basis in $L_{2}$. However, if we put aside the works which cover the

* E-mail: nazimkerimov@yahoo.com

† E-mail:mat-ufuk@hotmail.com 
block-basis property (or the basis property with bracket) of a system of root functions, see e.g. [28], then the basicity in $L_{p}, 1<p<\infty$, of the system of root functions of ordinary differential operators with not strongly regular boundary conditions has not been studied enough. An example of a differential operator with regular boundary conditions (but not strongly regular) whose root functions do not form a basis in the space $L_{2}$ was given in [14].

In 1976, N.I. Ionkin [11] studied a non-classic problem for heat conduction in a homogeneous matter. By separation of variables, this problem is reduced to the boundary value problem

$$
-y^{\prime \prime}=\lambda y, \quad y(0)=0, \quad y^{\prime}(0)=y^{\prime}(1)
$$

whose boundary conditions are regular but not strongly regular. All eigenvalues of this problem, beginning from the second one, are double multiples and the general number of associated functions is infinite. Nevertheless, it was established that the system of root functions of this problem chosen in a special way forms an unconditional basis in $L_{2}(0,1)$.

In [13], it was established that, under conditions $q(x) \in C^{(4)}[0,1]$ and $q(1)-q(0) \neq 0$, all eigenvalues of the differential operator generated by the expression $l(y)=y^{\prime \prime}+q(x) y, x \in(0,1)$, and periodic (antiperiodic) boundary conditions, starting from some number are simple and the root functions of this operator form an unconditional basis of $L_{2}$. Note that periodic and antiperiodic boundary conditions are regular, but not strongly regular.

A.S. Makin [16-21], P.B. Djakov and B.S. Mityagin [3-6] have investigated in detail some spectral properties of SturmLiouville operators with not strongly regular boundary conditions. The existence of a wide class of boundary value problems for second order ordinary differential operators with regular but not strongly regular boundary conditions, whose system of root functions does not form a basis in $L_{2}$ is established in the paper [16]. Some incisive results on the absence of the basis property were obtained in [3]. In particular, in [3] examples of potentials, with arbitrary smoothness such that the corresponding system of root functions does not contain a basis in $L_{2}$, are given.

It was proved in [17] that the system of root functions of the differential operator

$$
\left\{\begin{array}{l}
l(y)=y^{\prime \prime}+q(x) y, \\
y^{\prime}(1)-(-1)^{\sigma} y^{\prime}(0)+\gamma y(0)=0, \quad y(1)-(-1)^{\sigma} y(0)=0
\end{array}\right.
$$

forms an unconditional basis of the space $L_{2}(0,1)$, where $q(x)$ is an arbitrary complex-valued function from the class $L_{1}(0,1), \gamma$ is an arbitrary nonzero complex constant and $\sigma=0,1$. Under the condition $\gamma=0$ (periodic and antiperiodic boundary conditions) in $[4,18]$, necessary and sufficient conditions of unconditional basicity in $L_{2}(0,1)$ of the system of root functions of differential operator (1) are obtained in terms of the Fourier coefficients of the potential $q(x)$, see also $[8,15,22,23,25,29]$. Note that in [4], the class of potentials considered is much wider (the proof is given in [5]). Some other interesting results about Riesz basicity of root functions of such operators with trigonometric polynomial potentials were obtained in $[4,5]$. Moreover, recently, P.B. Djakov and B.S. Mityagin [6] proved a general criterion for basicity in terms of the Fourier coefficients of the potential (without any restriction on the class of potentials, even for distribution potentials). We can also refer to [2, 24, 30-32] where spectral properties of boundary-value problems for ordinary differential operators with regular boundary conditions (but not strongly regular) are studied.

One of the effective ways of investigation of spectral properties of differential operators is the method worked out by V.A. Ilin and his followers (V.D. Budaev, I.S. Lomov, V.M. Kurbanov, A.S. Makin and others). The present paper was stimulated by these investigations, see [10].

Henceforward, by $L$ we denote a differential operator generated by the differential expression

$$
l(y)=y^{\mathrm{iv}}+p_{2}(x) y^{\prime \prime}+p_{1}(x) y^{\prime}+p_{0}(x) y, \quad x \in(0,1)
$$

and the boundary conditions

$$
\begin{aligned}
& U_{s}(y) \equiv y^{(s)}(1)-(-1)^{\sigma} y^{(s)}(0)+\sum_{l=0}^{s-1} \alpha_{s, l} y^{(l)}(0)=0, \quad s=1,2,3, \\
& U_{0}(y) \equiv y(1)-(-1)^{\sigma} y(0)=0,
\end{aligned}
$$


where $p_{j}(x) \in L_{1}(0,1), j=0,1,2$, are complex-valued functions; $\alpha_{s, l}, s=1,2,3, l=\overline{0, s-1}$, are arbitrary complex constants; and $\sigma=0,1$. It is easy to verify that boundary conditions (3) are regular, but not strongly regular.

Usually, in order to investigate spectral properties of differential operators with not strongly regular boundary conditions, more exact asymptotic formulae for eigenvalues and eigenfunctions are required. In this paper, such asymptotic formulae for eigenvalues and eigenfunctions of the differential operator $L$ are established, and the basicity of the system of root functions of this operator is investigated in the space $L_{p}(0,1), 1<p<\infty$.

Before we formulate the basic results of this paper we introduce some notations. Assume that $W_{1}^{j}(0,1), j=1,2$, are Sobolev spaces, $W_{1}^{0}(0,1) \equiv L_{1}(0,1)$ and

$$
\begin{gathered}
c_{0}=\int_{0}^{1} p_{2}(\xi) d \xi, \\
d_{n}=\int_{0}^{1} p_{2}(\xi) e^{2(2 n-\sigma) \pi i \xi} d \xi, \quad d_{-n}=\int_{0}^{1} p_{2}(\xi) e^{-2(2 n-\sigma) \pi i \xi} d \xi, \\
\varepsilon_{n}=\left|d_{n}\right|+\left|d_{-n}\right|+n^{-1} .
\end{gathered}
$$

The following assertions are the basic results of this paper.

\section{Theorem 1.1.}

Let $p_{j}(x) \in L_{1}(0,1), j=0,1,2$, be arbitrary complex-valued functions and let $\alpha_{3,2}+\alpha_{1,0} \neq \alpha_{2,1}$. Then all eigenvalues of differential operator (2)-(3), except for a finite number, are simple and form two infinite sequences $\lambda_{n, 1}, n=1,2, \ldots$, and $\lambda_{n, 2}, n=1,2, \ldots$ Moreover, for sufficiently large numbers $n$, the asymptotic formulae

$$
\begin{aligned}
& \lambda_{n+n_{1}, 1}=((2 n-\sigma) \pi)^{4}\left\{1+\frac{2(-1)^{\sigma} \alpha_{2,1}-c_{0}}{((2 n-\sigma) \pi)^{2}}+O\left(n^{-2} \varepsilon_{n}\right)\right\}, \\
& \lambda_{n+n_{2}, 2}=((2 n-\sigma) \pi)^{4}\left\{1+\frac{2(-1)^{\sigma}\left(\alpha_{3,2}+\alpha_{1,0}\right)-c_{0}}{((2 n-\sigma) \pi)^{2}}+O\left(n^{-2} \varepsilon_{n}\right)\right\},
\end{aligned}
$$

are valid, where $n_{1}$ and $n_{2}$ are certain integers. Furthermore, for sufficiently large numbers $n$, the corresponding eigenfunctions $u_{n, 1}(x)$ and $u_{n, 2}(x), n=1,2, \ldots$, have the following asymptotic formulae:

$$
u_{n_{1}+n, 1}(x)=\sqrt{2} \sin (2 n-\sigma) \pi x+O\left(\varepsilon_{n}\right), \quad u_{n_{2}+n, 2}(x)=\sqrt{2} \cos (2 n-\sigma) \pi x+O\left(\varepsilon_{n}\right) .
$$

\section{Theorem 1.2.}

Let $p_{j}(x) \in W_{1}^{j}(0,1), j=0,1,2$, be arbitrary complex-valued functions and let $\alpha_{3,2}+\alpha_{1,0} \neq \alpha_{2,1}$. Then the system of root functions of differential operator (2)-(3) forms a basis in the space $L_{p}(0,1), 1<p<\infty$, and this basis is unconditional for $p=2$.

\section{Corollary 1.3.}

Let all conditions of Theorem 1.2 be fulfilled, and $n_{1}, n_{2}$ be integers from Theorem 1.1. Then $n_{1}+n_{2}=1-\sigma$ and we can choose $n_{1}=0$ and $n_{2}=1-\sigma$.

\section{Some auxiliary results}

Let

$$
S_{0}=\left\{\rho \in \mathbb{C}: 0 \leq \arg \rho \leq \frac{\pi}{4}\right\},
$$

where $\mathbb{C}$ is the set of complex numbers. We denote by $w_{k}, k=\overline{1,4}$, different 4 -th roots of -1 . It is known that, see [27, Chapter II, $\S 4.2]$, the numbers $w_{k}, k=\overline{1,4}$, can be ordered in such a way that for all $\rho \in S_{0}$ the inequalities

$$
\Re\left(\rho w_{1}\right) \leq \Re\left(\rho w_{2}\right) \leq \Re\left(\rho w_{3}\right) \leq \Re\left(\rho w_{4}\right)
$$


hold, where $\Re z$ means the real parts of $z$. Henceforward, the numbers $w_{k}, k=\overline{1,4}$, will be such that for all $\rho \in S_{0}$ the inequalities (10) are valid. It was proved that in this case the numbers $w_{k}, k=\overline{1,4}$, can be determined by means of equalities, see [27, Chapter II, §4.8],

$$
w_{1}=e^{3 \pi i / 4}, \quad w_{2}=e^{-3 \pi i / 4}, \quad w_{3}=e^{\pi i / 4}, \quad w_{4}=e^{-\pi i / 4} .
$$

It is easy to see that

$$
w_{1}=-w_{4}, \quad w_{2}=-w_{3} .
$$

Lemma 2.1.

In $S_{0}$ the following inequalities are valid:

$$
\Re\left(\rho w_{1}\right) \leq-\frac{\sqrt{2}}{2}|\rho|, \quad \Re\left(\rho w_{4}\right) \geq \frac{\sqrt{2}}{2}|\rho| .
$$

Proof. By (12), it suffices to prove the first inequality in (13). Since $\rho \in S_{0}$, it holds $\rho=|\rho| e^{i \theta \pi / 4}$ where $0 \leq \theta \leq 1$. Consequently, from (11)

$$
\Re\left(\rho w_{1}\right)=|\rho| \cdot \Re\left(e^{(3+\theta) \pi i / 4}\right)=-|\rho| \cdot \cos \frac{1-\theta}{4} \pi \leq-\frac{\sqrt{2}}{2}|\rho| .
$$

Consider the domain obtained from the section $S_{0}$, see (9), by a translation $\rho \mapsto \rho-c$, where $c$ is a fixed complex number. This new sector with its vertex at the point $\rho=-c$ will be denoted by $T_{0}$. Obviously, for the new section $T_{0}$ the inequalities (10) and (13) can be rewritten in the forms

$$
\begin{aligned}
& \Re\left((\rho+c) w_{1}\right) \leq \Re\left((\rho+c) w_{2}\right) \leq \Re\left((\rho+c) w_{3}\right) \leq \Re\left((\rho+c) w_{4}\right), \\
& \Re\left((\rho+c) w_{1}\right) \leq-\frac{\sqrt{2}}{2}|\rho+c|, \quad \Re\left((\rho+c) w_{4}\right) \geq \frac{\sqrt{2}}{2}|\rho+c| .
\end{aligned}
$$

Fix such a domain $T_{0}$. It is known, see [27, Chapter II, $\left.\S 4.5-4.6\right]$, that the equation

$$
l(y)+\rho^{4} y=0
$$

has, for the region $T_{0}$ of complex $\rho$-plane, four linearly independent solutions $y_{k}(x, \rho), k=\overline{1,4}$, which are regular for $\rho \in T_{0}$ with sufficiently large $|\rho|$, and which, with their derivatives satisfy the integro-differential equations

$$
\frac{d^{m} y_{k}(x, \rho)}{d x^{m}}=\rho^{m} w_{k}^{m} e^{\rho w_{k} x}+\frac{1}{4 \rho^{3}} \int_{0}^{x} \frac{\partial^{m} K_{1}(x, \xi, \rho)}{\partial x^{m}} M_{\xi}\left(y_{k}\right) d \xi-\frac{1}{4 \rho^{3}} \int_{x}^{1} \frac{\partial^{m} K_{2}(x, \xi, \rho)}{\partial x^{m}} M_{\xi}\left(y_{k}\right) d \xi, \quad m=\overline{0,3}
$$

where

$$
\begin{gathered}
K_{1}(x, \xi, \rho)=\sum_{\alpha=1}^{k} w_{\alpha} e^{\rho w_{\alpha}(x-\xi)}, \quad K_{2}(x, \xi, \rho)=\sum_{\alpha=k+1}^{4} w_{\alpha} e^{\rho w_{\alpha}(x-\xi)}, \\
M_{x}(y)=p_{2}(x) y^{\prime \prime}(x)+p_{1}(x) y^{\prime}(x)+p_{0}(x) y(x) .
\end{gathered}
$$

Moreover, it is known [27, Chapter II, § 4.5] that

$$
\frac{d^{m} y_{k}(x, \rho)}{d x^{m}}=\rho^{m} e^{\rho w_{k} x} z_{k, m}(x, \rho)
$$


where $z_{k, m}(x, \rho)$ is an analytic function of $\rho$ and it satisfies

$$
z_{k, m}(x, \rho)=w_{k}^{m}+O\left(\rho^{-1}\right), \quad k=\overline{1,4}, \quad m=\overline{0,3}
$$

From (17), (19) and (20), we have

$$
\begin{aligned}
z_{k, m}(x, \rho)=w_{k}^{m} & +\frac{1}{4 \rho} \int_{0}^{x} e^{-\rho w_{k}(x-\xi)} \rho^{-m} \frac{\partial^{m} K_{1}(x, \xi, \rho)}{\partial x^{m}} \sum_{j=0}^{2} \frac{p_{j}(\xi)}{\rho^{2-j}} z_{k, j}(\xi, \rho) d \xi \\
& -\frac{1}{4 \rho} \int_{x}^{1} e^{-\rho w_{k}(x-\xi)} \rho^{-m} \frac{\partial^{m} K_{2}(x, \xi, \rho)}{\partial x^{m}} \sum_{j=0}^{2} \frac{p_{j}(\xi)}{\rho^{2-j}} z_{k, j}(\xi, \rho) d \xi
\end{aligned}
$$

Taking into account expressions (18) of the functions $K_{1}(x, \xi, \rho)$ and $K_{2}(x, \xi, \rho)$, we have the representation

$$
\begin{aligned}
z_{k, m}(x, \rho)=w_{k}^{m}+\frac{w_{k}^{m+1}}{4 \rho} \int_{0}^{x} \sum_{j=0}^{2} \frac{p_{j}(\xi)}{\rho^{2-j}} z_{k, j}(\xi, \rho) d \xi & +\frac{1}{4 \rho} \sum_{\alpha=1}^{k-1} w_{\alpha}^{m+1} \int_{0}^{x} e^{\rho\left(w_{\alpha}-w_{k}\right)(x-\xi)} \sum_{j=0}^{2} \frac{p_{j}(\xi)}{\rho^{2-j}} z_{k, j}(\xi, \rho) d \xi \\
& -\frac{1}{4 \rho} \sum_{\alpha=k+1}^{4} w_{\alpha}^{m+1} \int_{x}^{1} e^{\rho\left(w_{\alpha}-w_{k}\right)(x-\xi)} \sum_{j=0}^{2} \frac{p_{j}(\xi)}{\rho^{2-j}} z_{k, j}(\xi, \rho) d \xi .
\end{aligned}
$$

Note that by (14) we have

$$
\Re\left(\rho\left(w_{\alpha}-w_{\beta}\right)\right)=\Re\left((\rho+c)\left(w_{\alpha}-w_{\beta}\right)\right)-\Re\left(c\left(w_{\alpha}-w_{\beta}\right)\right) \leq 2|c|,
$$

where $1 \leq \alpha \leq \beta \leq 4$. From here and (21) we obtain

$$
\int_{0}^{x} p_{j}(\xi) z_{k, j}(\xi, \rho) e^{\rho\left(w_{\alpha}-w_{k}\right)(x-\xi)} d \xi=O(1), \quad \alpha \leq k ; \quad \int_{x}^{1} p_{j}(\xi) z_{k, j}(\xi, \rho) e^{\rho\left(w_{\alpha}-w_{k}\right)(x-\xi)} d \xi=O(1), \quad \alpha \geq k
$$

where $k=\overline{1,4}$ and $j=0,1,2$. Consequently, it follows from (22) that

$$
\begin{aligned}
z_{k, m}(x, \rho)=w_{k}^{m} & +\frac{w_{k}^{m+1}}{4 \rho} \int_{0}^{x} \sum_{j=1}^{2} \frac{p_{j}(\xi)}{\rho^{2-j}} z_{k, j}(\xi, \rho) d \xi+\frac{1}{4 \rho} \sum_{\alpha=1}^{k-1} w_{\alpha}^{m+1} \int_{0}^{x} e^{\rho\left(w_{\alpha}-w_{k}\right)(x-\xi)} \sum_{j=1}^{2} \frac{p_{j}(\xi)}{\rho^{2-j}} z_{k, j}(\xi, \rho) d \xi \\
& -\frac{1}{4 \rho} \sum_{\alpha=k+1}^{4} w_{\alpha}^{m+1} \int_{x}^{1} e^{\rho\left(w_{\alpha}-w_{k}\right)(x-\xi)} \sum_{j=1}^{2} \frac{p_{j}(\xi)}{\rho^{2-j}} z_{k, j}(\xi, \rho) d \xi+O\left(\rho^{-3}\right) .
\end{aligned}
$$

Thus, in view of (23), the formulae

$$
\begin{aligned}
& z_{k, m}(0, \rho)=w_{k}^{m}-\frac{1}{4 \rho} \sum_{\alpha=k+1}^{4} w_{\alpha}^{m+1} B_{\alpha, k}(\rho)+O\left(\rho^{-3}\right), \\
& z_{k, m}(1, \rho)=w_{k}^{m}+\frac{w_{k}^{m+1}}{4 \rho} \sum_{j=1}^{2} \frac{1}{\rho^{2-j}} \int_{0}^{1} p_{j}(\xi) z_{k, j}(\xi, \rho) d \xi+\frac{1}{4 \rho} \sum_{\alpha=1}^{k-1} w_{\alpha}^{m+1} B_{\alpha, k}(\rho)+O\left(\rho^{-3}\right),
\end{aligned}
$$

are valid, where

$$
B_{\alpha, k}= \begin{cases}\sum_{j=1}^{2} \frac{1}{\rho^{2-j}} \int_{0}^{1} p_{j}(\xi) z_{k, j}(\xi, \rho) e^{-\rho\left(w_{\alpha}-w_{k}\right) \xi} d \xi & \text { if } 1 \leq k<\alpha \leq 4, \\ \sum_{j=1}^{2} \frac{1}{\rho^{2-j}} \int_{0}^{1} p_{j}(\xi) z_{k, j}(\xi, \rho) e^{\rho\left(w_{\alpha}-w_{k}\right)(1-\xi)} d \xi & \text { if } 1 \leq \alpha<k \leq 4 .\end{cases}
$$


It is easy to verify that for an arbitrary function $f(x) \in L_{1}(0,1)$,

$$
\int_{0}^{1} f(\xi) e^{\tau(1-\xi)} d \xi=o(1), \quad \int_{0}^{1} f(\xi) e^{\tau \xi} d \xi=o(1), \quad|\tau| \rightarrow+\infty
$$

hold for $\Re \tau \leq c_{1}=$ const. Hence, by virtue of (25) and (21) we have

$$
B_{\alpha, k}(\rho)=o(1), \quad \alpha \neq k
$$

A direct calculation using formulae (21), (24)-(25) shows that for $s=\overline{0,3}$ the following equalities are valid:

$$
\begin{aligned}
& z_{2, s}(0, \rho)=w_{2}^{s}-\frac{w_{4}^{s+1}}{4 \rho} B_{4,2}(\rho)-\frac{(-1)^{s} w_{2}^{s}}{4 \rho w_{2}} \int_{0}^{1} p_{2}(\xi) e^{2 \rho w_{2} \xi} d \xi+O\left(\rho^{-2}\right), \\
& z_{3, s}(0, \rho)=w_{3}^{s}-\frac{w_{4}^{s+1}}{4 \rho} B_{4,3}(\rho)+O\left(\rho^{-2}\right) \\
& z_{2, s}(1, \rho)=w_{2}^{s}-\frac{w_{2}^{s}}{4 \rho w_{2}} \int_{0}^{1} p_{2}(\xi) d \xi+\frac{w_{1}^{s+1}}{4 \rho} B_{1,2}(\rho)+O\left(\rho^{-2}\right), \\
& z_{3, s}(1, \rho)=w_{3}^{s}-\frac{w_{3}^{s}}{4 \rho w_{3}} \int_{0}^{1} p_{2}(\xi) d \xi+\frac{w_{1}^{s+1}}{4 \rho} B_{1,3}(\rho)+\frac{(-1)^{s} w_{3}^{s}}{4 \rho w_{3}} \int_{0}^{1} p_{2}(\xi) e^{-2 \rho w_{3}(1-\xi)} d \xi+O\left(\rho^{-2}\right) .
\end{aligned}
$$

\section{Proof of Theorem 1.1}

Let

$$
\Delta(\rho)=\left|\begin{array}{llll}
U_{3}\left(y_{1}\right) & U_{3}\left(y_{2}\right) & U_{3}\left(y_{3}\right) & U_{3}\left(y_{4}\right) \\
U_{2}\left(y_{1}\right) & U_{2}\left(y_{2}\right) & U_{2}\left(y_{3}\right) & U_{2}\left(y_{4}\right) \\
U_{1}\left(y_{1}\right) & U_{1}\left(y_{2}\right) & U_{1}\left(y_{3}\right) & U_{1}\left(y_{4}\right) \\
U_{0}\left(y_{1}\right) & U_{0}\left(y_{2}\right) & U_{0}\left(y_{3}\right) & U_{0}\left(y_{4}\right)
\end{array}\right|
$$

where $y_{k}(x, \rho), k=\overline{1,4}$, are the linearly independent solutions of the equation (16). It is known [27, Chapter II, $\left.\S 4.9\right]$ that if we properly choose the vertex $\rho=-c$ of the domain $T_{0}$, then the eigenvalues $\lambda$ of the differential operator (2)-(3) whose absolute values are sufficiently large have the form $\lambda=-\rho^{4}$, where the numbers $\rho$ are the roots of the equation

$$
\Delta(\rho)=0
$$

in the domain $T_{0}$, and the set of such points $\rho$ includes all roots of (29) in the domain $T_{0}$ except for a finite number. By (20) for $s=\overline{0,3}$ and $k=\overline{1,4}$ we have

$$
U_{s}\left(y_{k}\right)=\rho^{s}\left\{e^{\rho w_{k}} z_{k, s}(1, \rho)-(-1)^{\sigma} z_{k, s}(0, \rho)+\sum_{l=0}^{s-1} \frac{\alpha_{s, l}}{\rho^{s-l}} z_{k, l}(0, \rho)\right\}
$$

where $\alpha_{0,-1}=\alpha_{0,0}=0$. According to (15), $e^{\rho w_{1}}$ tends exponentially to zero and $e^{\rho w_{4}}$ tends exponentially to infinity. Consequently, by (30) and (21) the following equalities are valid:

$$
U_{s}\left(y_{1}\right)=-\rho^{s}\left\{(-1)^{\sigma} z_{1, s}(0, \rho)-\sum_{l=0}^{s-1} \frac{\alpha_{s, l}}{\rho^{s-l}} z_{1, l}(0, \rho)+O\left(\rho^{-3}\right)\right\}, \quad U_{s}\left(y_{4}\right)=\rho^{s} e^{\rho w_{4}}\left\{z_{4, s}(1, \rho)+O\left(\rho^{-3}\right)\right\}
$$


Let

$$
A_{s, k}(\rho)= \begin{cases}(-1)^{\sigma} z_{1, s}(0, \rho)-\sum_{l=0}^{s-1} \frac{\alpha_{s, l}}{\rho^{s-l}} z_{1, l}(0, \rho), & \text { if } k=1, \\ e^{\rho w_{k}} z_{k, s}(1, \rho)-(-1)^{\sigma} z_{k, s}(0, \rho)+\sum_{l=0}^{s-1} \frac{\alpha_{s, l}}{\rho^{s-l}} z_{k, l}(0, \rho), & \text { if } k=2,3, \\ z_{4, s}(1, \rho), & \text { if } k=4 .\end{cases}
$$

By virtue of (30)-(32) we have

$$
\begin{aligned}
& U_{s}\left(y_{1}\right)=-\rho^{s}\left\{A_{s, 1}(\rho)+O\left(\rho^{-3}\right)\right\}, \\
& U_{s}\left(y_{k}\right)=\rho^{s}\left\{A_{s, k}(\rho)+O\left(\rho^{-3}\right)\right\}, \quad k=2,3 \\
& U_{s}\left(y_{4}\right)=\rho^{s} e^{\rho w_{4}}\left\{A_{s, 4}(\rho)+O\left(\rho^{-3}\right)\right\} .
\end{aligned}
$$

We substitute these expressions in the equation (29), see (28), and divide out the common factors $\rho^{3}, \rho^{2}, \rho$ of the rows and also the common factor $e^{\rho w_{4}}$ of the last column of the determinant $\Delta(\rho)$. Then the equation (29) can be written in the form

$$
\Delta_{1}(\rho)+O\left(\rho^{-3}\right)=0
$$

where

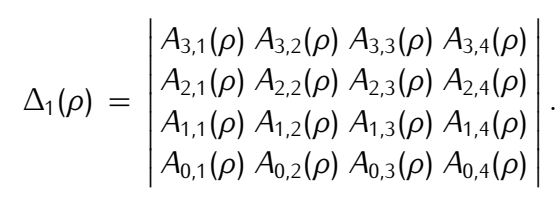

By using the formulae (47a) and (47b) in [27, Chapter II, $\S 4.9]$, we get that if $\rho$ is a root of equation (29) or (34), then

$$
e^{\rho w_{2}}-(-1)^{\sigma}=O\left(|\rho|^{-1 / 2}\right), \quad e^{\rho w_{3}}-(-1)^{\sigma}=O\left(|\rho|^{-1 / 2}\right) .
$$

From the relations (21), (32), (36) for $s=\overline{0,3}$ and $k=2,3$ we infer that

$$
\begin{array}{cc}
A_{s, 1}(\rho)=(-1)^{\sigma} w_{1}^{s}+O\left(\rho^{-1}\right), & A_{s, 4}(\rho)=w_{4}^{s}+O\left(\rho^{-1}\right), \\
A_{s, k}(\rho)=\left(e^{\rho w_{k}}-(-1)^{\sigma}\right) w_{k}^{s}+O\left(\rho^{-1}\right), & A_{s, k}(\rho)=O\left(|\rho|^{-1 / 2}\right) .
\end{array}
$$

From (37)-(38), we deduce that the equation (34) is equivalent to

$$
\left(e^{\rho w_{2}}-(-1)^{\sigma}\right)\left(e^{\rho w_{3}}-(-1)^{\sigma}\right) W_{0}+O\left(|\rho|^{-3 / 2}\right)=0
$$

where

$$
W_{0}=(-1)^{\sigma}\left|\begin{array}{cccc}
w_{1}^{3} & w_{2}^{3} & w_{3}^{3} & w_{4}^{3} \\
w_{1}^{2} & w_{2}^{2} & w_{3}^{2} & w_{4}^{2} \\
w_{1} & w_{2} & w_{3} & w_{4} \\
1 & 1 & 1 & 1
\end{array}\right|=-16(-1)^{\sigma}
$$

Since $w_{3}=-w_{2}$ and $e^{\rho w_{2}}=O(1)$, the equation (39) can be written in the form

$$
\left(e^{p w_{2}}-(-1)^{\sigma}\right)^{2}+O\left(|\rho|^{-3 / 2}\right)=0
$$

From this, we can easily get

$$
e^{\rho w_{2}}-(-1)^{\sigma}=O\left(|\rho|^{-3 / 4}\right), \quad e^{\rho w_{3}}-(-1)^{\sigma}=O\left(|\rho|^{-3 / 4}\right) .
$$


Note that, from (38) and (41) for $s=\overline{0,3}$ and $k=2,3$ it follows that

$$
A_{s, k}(\rho)=O\left(|\rho|^{-3 / 4}\right)
$$

By (35), (37) and (42), the equation (34) is equivalent to

$$
\Delta_{2}(\rho)+o\left(\rho^{-2}\right)=0
$$

where

$$
\Delta_{2}(\rho)=\left|\begin{array}{cccc}
(-1)^{\sigma} w_{1}^{3} & A_{3,2}(\rho) & A_{3,3}(\rho) & w_{4}^{3} \\
(-1)^{\sigma} w_{1}^{2} & A_{2,2}(\rho) & A_{2,3}(\rho) & w_{4}^{2} \\
(-1)^{\sigma} w_{1} & A_{1,2}(\rho) & A_{1,3}(\rho) & w_{4} \\
(-1)^{\sigma} & A_{0,2}(\rho) & A_{0,3}(\rho) & 1
\end{array}\right|
$$

According to the equality (32) for $s=\overline{0,3}$ and $k=2,3$ we have

$$
A_{s, k}(\rho)=e^{\rho w_{k}} z_{k, s}(1, \rho)-(-1)^{\sigma} z_{k, s}(0, \rho)+\frac{\alpha_{s, s-1}}{\rho} z_{k, s-1}(0, \rho)+O\left(\rho^{-2}\right) .
$$

From here and (26)-(27) it follows

$$
A_{s, k}(\rho)=A_{s, k}^{(k)}(\rho)+B_{s, k}^{(k)}(\rho)+O\left(\rho^{-2}\right), \quad k=2,3,
$$

where

$$
\begin{gathered}
A_{s, 2}^{(2)}(\rho)=w_{2}^{s}\left\{e^{\rho w_{2}}\left(1-\frac{c_{0}}{4 \rho w_{2}}\right)-(-1)^{\sigma}\left(1-\frac{(-1)^{s}}{4 \rho w_{2}} \gamma_{2}(\rho)\right)+\frac{\alpha_{s, s-1}}{\rho w_{2}}\right\}, \\
A_{s, 3}^{(3)}(\rho)=w_{3}^{s}\left\{e^{\rho w_{3}}\left(1-\frac{c_{0}}{4 \rho w_{3}}+\frac{(-1)^{s}}{4 \rho w_{3}} \gamma_{3}(\rho)\right)-(-1)^{\sigma}+\frac{\alpha_{s, s-1}}{\rho w_{3}}\right\}, \\
B_{s, 2}^{(2)}(\rho)=\frac{w_{1}^{s+1} e^{\rho w_{2}}}{4 \rho} B_{1,2}(\rho)+\frac{(-1)^{\sigma} w_{4}^{s+1}}{4 \rho} B_{4,2}(\rho), \quad B_{s, 3}^{(3)}(\rho)=\frac{w_{1}^{s+1} e^{\rho w_{3}}}{4 \rho} B_{1,3}(\rho)+\frac{(-1)^{\sigma} w_{4}^{s+1}}{4 \rho} B_{4,3}(\rho), \\
\gamma_{2}(\rho)=\int_{0}^{1} p_{2}(\xi) e^{2 \rho w_{2} \xi} d \xi, \quad \gamma_{3}(\rho)=\int_{0}^{1} p_{2}(\xi) e^{-2 \rho w_{3}(1-\xi)} d \xi
\end{gathered}
$$

and $c_{0}$ is the number defined in (4). Taking into account (47), it is easy to see that for $k=2,3$ the column $\left(B_{3, k}^{(k)}(\rho), B_{2, k}^{(k)}(\rho), B_{1, k}^{(k)}(\rho), B_{0, k}^{(k)}(\rho)\right)^{\top}$ is a linear combination of the first and last columns of the determinant $\Delta_{2}(\rho)$. From this and the asymptotic estimate

$$
A_{s, k}^{(k)}(\rho)=O\left(|\rho|^{-3 / 4}\right)
$$

it follows that the equation (43), see (44), is equivalent to

$$
\Delta_{3}(\rho)+o\left(\rho^{-2}\right)=0
$$

where

$$
\Delta_{3}(\rho)=\left|\begin{array}{cccc}
(-1)^{\sigma} w_{1}^{3} & A_{3,2}^{(2)}(\rho) & A_{3,3}^{(3)}(\rho) & w_{4}^{3} \\
(-1)^{\sigma} w_{1}^{2} & A_{2,2}^{(2)}(\rho) & A_{2,3}^{(3)}(\rho) & w_{4}^{2} \\
(-1)^{\sigma} w_{1} & A_{1,2}^{(2)}(\rho) & A_{1,3}^{(3)}(\rho) & w_{4} \\
(-1)^{\sigma} & A_{0,2}^{(2)}(\rho) & A_{0,3}^{(3)}(\rho) & 1
\end{array}\right|
$$


Spectral properties of some regular boundary value problems for fourth order differential operators

Let

$$
\begin{gathered}
A_{k}(\rho)=e^{\rho w_{k}}\left(1-\frac{c_{0}}{4 \rho w_{k}}\right)-(-1)^{\sigma}, \quad k=2,3, \\
E_{s, 2}(\rho)=4 \alpha_{s, s-1}+(-1)^{\sigma+s} \gamma_{2}(\rho), \quad E_{s, 3}(\rho)=4 \alpha_{s, s-1}+(-1)^{s} e^{\rho w_{3}} \gamma_{3}(\rho) .
\end{gathered}
$$

Hence, by (46) for $s=\overline{0,3}$ and $k=2,3$ we have

$$
A_{s, k}^{(k)}(\rho)=w_{k}^{s} A_{k}(\rho)+w_{k}^{s} \frac{E_{s, k}(\rho)}{4 \rho w_{k}} .
$$

We set

$$
\begin{gathered}
\Delta^{(1)}(\rho)=\left|\begin{array}{cccc}
(-1)^{\sigma} w_{1}^{3} & w_{2}^{3} & w_{3}^{3} E_{3,3}(\rho) & w_{4}^{3} \\
(-1)^{\sigma} w_{1}^{2} & w_{2}^{2} & w_{3}^{2} E_{2,3}(\rho) & w_{4}^{2} \\
(-1)^{\sigma} w_{1} & w_{2} & w_{3} E_{1,3}(\rho) & w_{4} \\
(-1)^{\sigma} & 1 & E_{0,3}(\rho) & 1
\end{array}\right|, \quad \Delta^{(2)}(\rho)=\left|\begin{array}{cccc}
(-1)^{\sigma} w_{1}^{3} & w_{2}^{3} E_{3,2}(\rho) & w_{3}^{3} & w_{4}^{3} \\
(-1)^{\sigma} w_{1}^{2} & w_{2}^{2} E_{2,2}(\rho) & w_{3}^{2} & w_{4}^{2} \\
(-1)^{\sigma} w_{1} & w_{2} E_{1,2}(\rho) & w_{3} & w_{4} \\
(-1)^{\sigma} & E_{0,2}(\rho) & 1 & 1
\end{array}\right|, \\
\Delta^{(3)}(\rho)=\left|\begin{array}{ccccc}
(-1)^{\sigma} w_{1}^{3} & w_{2}^{3} E_{3,2}(\rho) & w_{3}^{3} E_{3,3}(\rho) & w_{4}^{3} \\
(-1)^{\sigma} w_{1}^{2} & w_{2}^{2} E_{2,2}(\rho) & w_{3}^{2} E_{2,3}(\rho) & w_{4}^{2} \\
(-1)^{\sigma} w_{1} & w_{2} E_{1,2}(\rho) & w_{3} E_{1,3}(\rho) & w_{4} \\
(-1)^{\sigma} & E_{0,2}(\rho) & E_{0,3}(\rho) & 1
\end{array}\right|
\end{gathered}
$$

From (51), (54)-(56) it follows

$$
\Delta_{3}(\rho)=A_{2}(\rho) A_{3}(\rho) W_{0}+\frac{A_{2}(\rho)}{4 \rho w_{3}} \Delta^{(1)}(\rho)+\frac{A_{3}(\rho)}{4 \rho w_{2}} \Delta^{(2)}(\rho)-\frac{\Delta^{(3)}(\rho)}{\left(4 \rho w_{2}\right)^{2}}
$$

where $W_{0}$ is a determinant defined by the equality (40). Note that the last term in (57) is $O\left(\rho^{-2}\right)$. A direct calculation using (11) shows that

$$
\Delta^{(1)}(\rho)=\Delta^{(2)}(\rho)=-16(-1)^{\sigma}\left(\alpha_{3,2}+\alpha_{2,1}+\alpha_{1,0}\right)
$$

From here and (57) we deduce

$$
\Delta_{3}(\rho)=-16(-1)^{\sigma}\left[A_{2}(\rho) A_{3}(\rho)+\frac{\alpha_{3,2}+\alpha_{2,1}+\alpha_{1,0}}{4 \rho w_{2}}\left(A_{3}(\rho)-A_{2}(\rho)\right)\right]+O\left(\rho^{-2}\right) .
$$

Consequently, the equation (50) is equivalent to

$$
A_{1}(\rho) A_{2}(\rho)+\frac{\alpha_{3,2}+\alpha_{2,1}+\alpha_{1,0}}{4 \rho w_{2}}\left(A_{3}(\rho)-A_{2}(\rho)\right)+O\left(\rho^{-2}\right)=0 .
$$

Taking into account (52), we can rewrite the last equation in the form

$$
\left[e^{\rho w_{2}}\left(1-\frac{c_{0}}{4 \rho w_{2}}\right)-(-1)^{\sigma}\right]\left[e^{-\rho w_{2}}\left(1+\frac{c_{0}}{4 \rho w_{2}}\right)-(-1)^{\sigma}\right]+\frac{\alpha_{3,2}+\alpha_{2,1}+\alpha_{1,0}}{4 \rho w_{2}}\left[e^{-\rho w_{2}}-e^{\rho w_{2}}\right]+O\left(\rho^{-2}\right)=0
$$

or more precisely

$$
\left[1+\frac{\gamma}{4 \rho w_{2}}\right] e^{2 \rho w_{2}}-2(-1)^{\sigma} e^{\rho w_{2}}+\left[1-\frac{\gamma}{4 \rho w_{2}}+O\left(\rho^{-2}\right)\right]=0,
$$

102 
where $\gamma=(-1)^{\sigma}\left(\alpha_{3,2}+\alpha_{2,1}+\alpha_{1,0}\right)-c_{0}$. After some simplifications, the given equation is reduced to the form

$$
e^{2 \rho w_{2}}-2(-1)^{\sigma}\left(1-\frac{\gamma}{4 \rho w_{2}}\right) e^{\rho w_{2}}+1-\frac{\gamma}{2 \rho w_{2}}+O\left(\rho^{-2}\right)=0
$$

Hence, we obtain

$$
\left[e^{\rho w_{2}}-(-1)^{\sigma}\left(1-\frac{\gamma}{4 \rho w_{2}}\right)\right]^{2}+O\left(\rho^{-2}\right)=0
$$

It is easy to see that if $\rho \in T_{0}$ is a root of equation (59), then

$$
e^{\rho w_{2}}-(-1)^{\sigma}=O\left(\rho^{-1}\right)
$$

Obviously, the following equality also holds:

$$
e^{\rho w_{3}}-(-1)^{\sigma}=O\left(\rho^{-1}\right)
$$

From the last two relations and (38), for $s=\overline{0,3}$ and $k=2,3$ we have

$$
A_{s, k}(\rho)=O\left(\rho^{-1}\right)
$$

Consequently, the equation (34) is equivalent to

$$
\Delta_{2}(\rho)+O\left(\rho^{-3}\right)=0
$$

where $\Delta_{2}(\rho)$ is the determinant defined by (44).

We proved above that (43) is equivalent to (50) using (49). Repeating the same reasonings and using relation (60) instead of (49), we conclude that (61) is equivalent to

$$
\Delta_{3}(\rho)+O\left(\rho^{-3}\right)=0
$$

where $\Delta_{3}(\rho)$ is the determinant defined by (51). We use the representation (57) for $\Delta_{3}(\rho)$. We have $e^{\rho w_{3}}=(-1)^{\sigma}+O\left(\rho^{-1}\right)$. Then, by (53), we get for $s=\overline{0,3}$ and $k=2,3, E_{s, k}(\rho)=4 \alpha_{s, s-1}+(-1)^{\sigma+s} \gamma_{k}(\rho)+O\left(\rho^{-1}\right)$. After substituting these expressions in (56) and simple transformations we have

$$
\Delta^{(3)}(\rho)=-64(-1)^{\sigma} \alpha_{2,1}\left(\alpha_{3,2}+\alpha_{1,0}\right)+16\left(\gamma_{2}(\rho)+\gamma_{3}(\rho)\right)\left(\alpha_{3,2}-\alpha_{2,1}+\alpha_{1,0}\right)+16(-1)^{\sigma} \gamma_{2}(\rho) \gamma_{3}(\rho)+O\left(\rho^{-1}\right)
$$

From here, (57) and (58) we deduce that

$$
\Delta_{3}(\rho)=-16(-1)^{\sigma}\left\{A_{2}(\rho) A_{3}(\rho)+\frac{\delta_{0}}{4 \rho w_{2}}\left(A_{3}(\rho)-A_{2}(\rho)\right)-\frac{4 \delta_{1}}{\left(4 \rho w_{2}\right)^{2}}+O\left(\rho^{-2} \varepsilon(\rho)\right)\right\}
$$

where

$$
\begin{gathered}
\delta_{0}=\alpha_{3,2}+\alpha_{2,1}+\alpha_{1,0}, \quad \delta_{1}=\alpha_{2,1}\left(\alpha_{3,2}+\alpha_{1,0}\right), \\
\varepsilon(\rho)=\left|\gamma_{2}(\rho)+\gamma_{3}(\rho)\right|+\left|\gamma_{2}(\rho) \gamma_{3}(\rho)\right|+\left|\rho^{-1}\right| .
\end{gathered}
$$

Consequently, (62) is equivalent to

$$
A_{2}(\rho) A_{3}(\rho)+\frac{\delta_{0}}{4 \rho w_{2}}\left(A_{3}(\rho)-A_{2}(\rho)\right)-\frac{4 \delta_{1}}{\left(4 \rho w_{2}\right)^{2}}+O\left(\rho^{-2} \varepsilon(\rho)\right)=0
$$


Thus, according to (52), (62) is equivalent to

$$
\begin{aligned}
\left\{e^{\rho w}\left(1-\frac{c_{0}}{4 \rho w}\right)-(-1)^{\sigma}\right\} & \left\{e^{-\rho w}\left(1+\frac{c_{0}}{4 \rho w}\right)-(-1)^{\sigma}\right\} \\
& +\frac{\delta_{0}}{4 \rho w}\left\{e^{-\rho w}\left(1+\frac{c_{0}}{4 \rho w}\right)-e^{\rho w}\left(1-\frac{c_{0}}{4 \rho w}\right)\right\}-\frac{4 \delta_{1}}{(4 \rho w)^{2}}+O\left(\rho^{-2} \varepsilon(\rho)\right)=0
\end{aligned}
$$

where $w=w_{2}$. After some simplifications the given equation is reduced to the form

$$
\left(1-\frac{c_{0}}{4 \rho w}\right)\left(1+\frac{(-1)^{\sigma} \delta_{0}}{4 \rho w}\right) e^{2 \rho w}-2(-1)^{\sigma}\left(1-\frac{c_{0}^{2}+4 \delta_{1}}{2(4 \rho w)^{2}}\right) e^{\rho w}+\left(1+\frac{c_{0}}{4 \rho w}\right)\left(1-\frac{(-1)^{\sigma} \delta_{0}}{4 \rho w}\right)+O\left(\rho^{-2} \varepsilon(\rho)\right)=0
$$

The last equation splits into two equations:

$$
\begin{aligned}
& e^{\rho w}=(-1)^{\sigma}+\frac{(-1)^{\sigma} c_{0}-2 \alpha_{2,1}}{4 \rho w}+O\left(\rho^{-1} \varepsilon(\rho)\right), \\
& e^{\rho w}=(-1)^{\sigma}+\frac{(-1)^{\sigma} c_{0}-2\left(\alpha_{3,2}+\alpha_{1,0}\right)}{4 \rho w}+O\left(\rho^{-1} \varepsilon(\rho)\right) .
\end{aligned}
$$

We will investigate the equation (64). By using the Roushe theorem, it can be proved in a standard way [27, Chapter II, $\S 4.9]$ that the roots $\rho \in T_{0}$ of (64) whose absolute values are sufficiently large, lie in the domains $G_{n} \subset T_{0}, n=n_{0}, n_{0}+1, \ldots$, where $G_{n}$ is the $O\left(n^{-1}\right)$-neigbourhood of the point $-(2 n-\sigma) \pi i / w$ and $n_{0}$ is a sufficiently large natural number. Moreover, (64) has a unique root within each $G_{n}, n=n_{0}, n_{0}+1, \ldots$ The roots $\rho \in T_{0}$ of the equation (64) whose absolute values are sufficiently large satisfy the relation

$$
e^{\rho w}=(-1)^{\sigma}+O\left(\rho^{-1}\right)
$$

Let $\widetilde{\rho}$ be the root of equation (64) belonging to $G_{n}$. It is obvious from (66) that

$$
\begin{aligned}
\tilde{\rho} & =-\frac{(2 n-\sigma) \pi i}{w}+r \\
r & =O\left(n^{-1}\right) .
\end{aligned}
$$

Suppose that $d_{n}$ and $d_{-n}$ are numbers defined by (5). According to (48), (67) and (68) we have

$$
\gamma_{2}(\widetilde{\rho})=d_{-n}+O\left(n^{-1}\right), \quad \gamma_{3}(\widetilde{\rho})=d_{n}+O\left(n^{-1}\right)
$$

From here and (63) we deduce

$$
\varepsilon(\widetilde{\rho})=O\left(\varepsilon_{n}\right)
$$

where $\varepsilon_{n}$ is the number defined by (6).

Revise the form of $r$. From (67) it follows

$$
(\widetilde{\rho} w)^{-1}=\frac{-1}{(2 n-\sigma) \pi i}+O\left(n^{-3}\right), \quad e^{\tilde{\rho} w}=(-1)^{\sigma}\left\{1+r w+O\left(n^{-2}\right)\right\}
$$

Writing $\rho=\tilde{\rho}$ in (64) and using the relations (69)-(70), after simple transformations we have

$$
r=\frac{2(-1)^{\sigma} \alpha_{2,1}-c_{0}}{4 w(2 n-\sigma) \pi i}+O\left(n^{-1} \varepsilon_{n}\right)
$$


Thus, by (67)-(71), within $O\left(n^{-1}\right)$-neigbourhood $G_{n}$ of the point $z_{n}=-(2 n-\sigma) \pi i / w, n=n_{0}, n_{0}+1, \ldots$, equation (64) has the unique root

$$
\tilde{\rho}_{n, 1}=-\frac{1}{w}\left\{(2 n-\sigma) \pi i-\frac{2(-1)^{\sigma} \alpha_{2,1}-c_{0}}{4(2 n-\sigma) \pi i}\right\}+O\left(n^{-1} \varepsilon_{n}\right)
$$

Similarly we find that, within $O\left(n^{-1}\right)$-neigbourhood $G_{n}$ of the point $z_{n}, n=n_{0}, n_{0}+1, \ldots$, equation (65) has the unique root

$$
\tilde{\rho}_{n, 2}=-\frac{1}{w}\left\{(2 n-\sigma) \pi i-\frac{2(-1)^{\sigma}\left(\alpha_{3,2}+\alpha_{1,0}\right)-c_{0}}{4(2 n-\sigma) \pi i}\right\}+O\left(n^{-1} \varepsilon_{n}\right) .
$$

We seek for the eigenfunction $\widetilde{u}_{n, 1}(x)$, corresponding to the eigenvalue $\lambda=-\left(\widetilde{\rho}_{n, 1}\right)^{4}$ for sufficiently large $n$, in the form

$$
\widetilde{u}_{n, 1}(x)=\frac{-(-1)^{\sigma} \rho^{-5} e^{-\rho w_{4}} \sqrt{2}}{4 w_{2}\left(\alpha_{3,2}-\alpha_{2,1}+\alpha_{1,0}\right)}\left|\begin{array}{cccc}
y_{1}(x, \rho) & y_{2}(x, \rho) & y_{3}(x, \rho) & y_{4}(x, \rho) \\
U_{3}\left(y_{1}\right) & U_{3}\left(y_{2}\right) & U_{3}\left(y_{3}\right) & U_{3}\left(y_{4}\right) \\
U_{2}\left(y_{1}\right) & U_{2}\left(y_{2}\right) & U_{2}\left(y_{3}\right) & U_{2}\left(y_{4}\right) \\
U_{1}\left(y_{1}\right) & U_{1}\left(y_{2}\right) & U_{1}\left(y_{3}\right) & U_{1}\left(y_{4}\right)
\end{array}\right|_{\rho=\tilde{\rho}_{n, 1}}
$$

or more precisely

$$
\widetilde{u}_{n, 1}(x)=\frac{(-1)^{\sigma} \rho \sqrt{2}}{4 w_{2}\left(\alpha_{3,2}-\alpha_{2,1}+\alpha_{1,0}\right)}\left|\begin{array}{cccc}
-y_{1}(x, \rho) & y_{2}(x, \rho) & y_{3}(x, \rho) & e^{-\rho w_{4}} y_{4}(x, \rho) \\
-\rho^{-3} U_{3}\left(y_{1}\right) & \rho^{-3} U_{3}\left(y_{2}\right) & \rho^{-3} U_{3}\left(y_{3}\right) & \rho^{-3} e^{-\rho w_{4}} U_{3}\left(y_{4}\right) \\
-\rho^{-2} U_{2}\left(y_{1}\right) & \rho^{-2} U_{2}\left(y_{2}\right) & \rho^{-2} U_{2}\left(y_{3}\right) & \rho^{-2} e^{-\rho w_{4}} U_{2}\left(y_{4}\right) \\
-\rho^{-1} U_{1}\left(y_{1}\right) & \rho^{-1} U_{1}\left(y_{2}\right) & \rho^{-1} U_{1}\left(y_{3}\right) & \rho^{-1} e^{-\rho w_{4}} U_{1}\left(y_{4}\right)
\end{array}\right|_{\rho=\tilde{\rho}_{n, 1}}
$$

Henceforward, for simplicity of notation, we will write $\rho$ and $\varepsilon$ instead of $\tilde{\rho}_{n, 1}$ and $\varepsilon_{n}$, respectively. Since

$$
y_{k}(x, \rho)=O(1), \quad k=1,2,3, \quad e^{-\rho w_{4}} y_{4}(x, \rho)=O(1)
$$

in view of (74) and (33) we have

$$
\widetilde{u}_{n, 1}(x)=\frac{(-1)^{\sigma} \rho \sqrt{2}}{4 w_{2}\left(\alpha_{3,2}-\alpha_{2,1}+\alpha_{1,0}\right)}\left|\begin{array}{rrrr}
-y_{1}(x, \rho) & y_{2}(x, \rho) & y_{3}(x, \rho) & e^{-\rho w_{4}} y_{4}(x, \rho) \\
A_{3,1}(\rho) & A_{3,2}(\rho) & A_{3,3}(\rho) & A_{3,4}(\rho) \\
A_{2,1}(\rho) & A_{2,2}(\rho) & A_{2,3}(\rho) & A_{2,4}(\rho) \\
A_{1,1}(\rho) & A_{1,2}(\rho) & A_{1,3}(\rho) & A_{1,4}(\rho)
\end{array}\right|_{\rho=\tilde{\rho}_{n, 1}}+O\left(\rho^{-2}\right)
$$

Taking into account (37), (60), (75) and (76) we have

$$
\widetilde{u}_{n, 1}(x)=\frac{\rho \sqrt{2}}{4 \omega_{2}\left(\alpha_{3,2}-\alpha_{2,1}+\alpha_{1,0}\right)}\left[y_{3}(x, \rho) E_{2}(\rho)-y_{2}(x, \rho) E_{3}(\rho)\right]+O\left(\rho^{-1}\right),
$$

where

$$
E_{k}(\rho)=\left|\begin{array}{lll}
w_{1}^{3} & A_{3, k}(\rho) & w_{4}^{3} \\
w_{1}^{2} & A_{2, k}(\rho) & w_{4}^{2} \\
w_{1} & A_{1, k}(\rho) & w_{4}
\end{array}\right|, \quad k=2,3
$$

From here and (45) we obtain

$$
E_{k}(\rho)=\left|\begin{array}{lll}
w_{1}^{3} & A_{3, k}^{(k)}(\rho) & w_{4}^{3} \\
w_{1}^{2} & A_{2, k}^{(k)}(\rho) & w_{4}^{2} \\
w_{1} & A_{1, k}^{(k)}(\rho) & w_{4}
\end{array}\right|+\left|\begin{array}{lll}
w_{1}^{3} & B_{3, k}^{(k)}(\rho) & w_{4}^{3} \\
w_{1}^{2} & B_{2, k}^{(k)}(\rho) & w_{4}^{2} \\
w_{1} & B_{1, k}^{(k)}(\rho) & w_{4}
\end{array}\right|+O\left(\rho^{-2}\right)
$$


where $k=2,3$. According to (47), the last determinant is equal to zero. Consequently,

$$
E_{k}(\rho)=\left|\begin{array}{lll}
w_{1}^{3} & A_{3, k}^{(k)}(\rho) & w_{4}^{3} \\
w_{1}^{2} & A_{2, k}^{(k)}(\rho) & w_{4}^{2} \\
w_{1} & A_{1, k}^{(k)}(\rho) & w_{4}
\end{array}\right|+O\left(\rho^{-2}\right)
$$

Because of (46), for $s=\overline{0,3}$ and $k=2,3$ we have

$$
A_{s, k}^{(k)}(\rho)=w_{k}^{s}\left\{e^{\rho w_{k}}\left(1-\frac{c_{0}}{4 \rho w_{k}}\right)-(-1)^{\sigma}+\frac{\alpha_{s, s-1}}{\rho w_{k}}\right\}+O\left(\rho^{-1} \varepsilon\right) .
$$

From here and (72), after necessary simplifications it follows

$$
A_{s, k}^{(k)}(\rho)=w_{k}^{s} \frac{2 \alpha_{s, s-1}-\alpha_{2,1}}{2 \rho w k}+O\left(\rho^{-1} \varepsilon\right)
$$

where $s=\overline{0,3}$ and $k=2,3$. Further, from (78) and (79) we have

$$
E_{k}(\rho)=\frac{2 w_{1}}{\rho}\left(\alpha_{3,2}-\alpha_{2,1}+\alpha_{1,0}\right)+O\left(\rho^{-1} \varepsilon\right), \quad k=2,3
$$

Consequently, from (77) we obtain

$$
\widetilde{u}_{n, 1}(x)=\frac{\sqrt{2}}{2 i}\left(y_{3}(x, \rho)-y_{2}(x, \rho)\right)+O(\varepsilon),
$$

or more precisely

$$
\widetilde{u}_{n, 1}(x)=\frac{\sqrt{2}}{2 i}\left\{y_{3}\left(x, \widetilde{\rho}_{n, 1}\right)-y_{2}\left(x, \tilde{\rho}_{n, 1}\right)\right\}+O\left(\varepsilon_{n}\right) .
$$

On the other hand, from (20), (21) and (72) we deduce

$$
y_{2}\left(x, \tilde{\rho}_{n, 1}\right)=e^{-(2 n-\sigma) \pi i x}+O\left(n^{-1}\right), \quad y_{3}\left(x, \tilde{\rho}_{n, 1}\right)=e^{(2 n-\sigma) \pi i x}+O\left(n^{-1}\right) .
$$

Taking into account these expressions and (80) we have the representation

$$
\widetilde{u}_{n, 1}(x)=\sqrt{2} \sin (2 n-\sigma) \pi x+O\left(\varepsilon_{n}\right)
$$

We seek for the eigenfunction $\widetilde{u}_{n, 2}(x)$, corresponding to the eigenvalue $\lambda=-\widetilde{\rho}_{n, 2}^{4}$ for sufficiently large $n$, in the form

$$
\widetilde{u}_{n, 2}(x)=\frac{-(-1)^{\sigma} \rho^{-2} e^{-\rho w_{4}} \sqrt{2}}{4\left(\alpha_{3,2}-\alpha_{2,1}+\alpha_{1,0}\right)}\left|\begin{array}{cccc}
y_{1}(x, \rho) & y_{2}(x, \rho) & y_{3}(x, \rho) & y_{4}(x, \rho) \\
U_{2}\left(y_{1}\right) & U_{2}\left(y_{2}\right) & U_{2}\left(y_{3}\right) & U_{2}\left(y_{4}\right) \\
U_{1}\left(y_{1}\right) & U_{1}\left(y_{2}\right) & U_{1}\left(y_{3}\right) & U_{1}\left(y_{4}\right) \\
U_{0}\left(y_{1}\right) & U_{0}\left(y_{2}\right) & U_{0}\left(y_{3}\right) & U_{0}\left(y_{4}\right)
\end{array}\right|_{\rho=\tilde{\rho}_{n, 2}} .
$$

By a similar reasoning (see the above case for the eigenfunction $\widetilde{u}_{n, 1}(x)$ ), we get

$$
\tilde{u}_{n, 2}(x)=\sqrt{2} \cos (2 n-\sigma) \pi x+O\left(\varepsilon_{n}\right)
$$

Thus, it is established (see the formulae (72) and (73)) that there exist two infinite sequences of simple eigenvalues

$$
\begin{aligned}
& \lambda_{n_{0}}^{\prime}, \lambda_{n_{0}+1}^{\prime}, \lambda_{n_{0}+2}^{\prime}, \ldots \\
& \lambda_{n_{0}}^{\prime \prime}, \lambda_{n_{0}+1}^{\prime \prime}, \lambda_{n_{0}+2}^{\prime \prime}, \ldots
\end{aligned}
$$


and the asymptotic formulae

$$
\begin{aligned}
& \lambda_{n}^{\prime}=-\tilde{\rho}_{n, 1}^{4}=((2 n-\sigma) \pi)^{4}\left\{1+\frac{2(-1)^{\sigma} \alpha_{2,1}-c_{0}}{((2 n-\sigma) \pi)^{2}}+O\left(n^{-2} \varepsilon_{n}\right)\right\} \\
& \lambda_{n}^{\prime \prime}=-\tilde{\rho}_{n, 2}^{4}=((2 n-\sigma) \pi)^{4}\left\{1+\frac{2(-1)^{\sigma}\left(\alpha_{3,2}+\alpha_{1,0}\right)-c_{0}}{((2 n-\sigma) \pi)^{2}}+O\left(n^{-2} \varepsilon_{n}\right)\right\}
\end{aligned}
$$

are valid. Besides eigenvalues (83) and (84) there can exist only finitely many eigenvalues counted with multiplicities.

Assume that besides eigenvalues (83) and (84) the differential operator $L$ has $m$ eigenvalues counted with multiplicities Let $m=m_{1}+m_{2}$, where $m_{1}$ and $m_{2}$ are arbitrary nonnegative integers. Add $m_{1}\left(m_{2}\right)$ numbers from the remaining $m$ eigenvalues to the sequence (83) (respectively (84)). We get two sequences of the form

$$
c_{1}, c_{2}, \ldots, c_{m_{1}}, \lambda_{n_{0}}^{\prime}, \lambda_{n_{0}+1}^{\prime}, \lambda_{n_{0}+2}^{\prime}, \ldots, \quad e_{1}, e_{2}, \ldots, e_{m_{1}}, \lambda_{n_{0}}^{\prime \prime}, \lambda_{n_{0}+1}^{\prime \prime}, \lambda_{n_{0}+2}^{\prime \prime}, \ldots
$$

Denote these sequences of eigenvalues by $\lambda_{1,1}, \lambda_{2,1}, \ldots, \lambda_{n, 1}, \ldots$ and $\lambda_{1,2}, \lambda_{2,2}, \ldots, \lambda_{n, 2}, \ldots$, respectively. It is easy to see that

$$
\lambda_{n+n_{1}, 1}=\lambda_{n}^{\prime}, \quad \lambda_{n+n_{2}, 2}=\lambda_{n}^{\prime \prime}, \quad n \geq n_{0},
$$

where $n_{1}=m_{1}-n_{0}+1$ and $n_{2}=m_{2}-n_{0}+1$. The formulae (7) are directly obtained from (85)-(87). In a parallel way, repeating similar reasoning with appropriate sequences of root functions, the asymptotic formulae (8) are obtained from (81) and (82).

\section{Proofs of Theorem 1.2 and Corollary 1.3}

Since $p_{2}(x) \in W_{1}^{2}(0,1)$, then according to (5) and (6) we have $\varepsilon_{n}=O\left(n^{-1}\right)$. Consequently, in this case, the asymptotic formulae (8) can be rewritten as

$$
u_{n_{1}+n, 1}(x)=\sqrt{2} \sin (2 n-\sigma) \pi x+O\left(n^{-1}\right), \quad u_{n_{2}+n, 2}(x)=\sqrt{2} \cos (2 n-\sigma) \pi x+O\left(n^{-1}\right) .
$$

Let

$$
v_{1,1}(x), v_{1,2}(x), \ldots, v_{n, 1}(x), v_{n, 2}(x), \ldots
$$

be the system which is biorthogonally conjugate to the system

$$
u_{1,1}(x), u_{1,2}(x), \ldots, u_{n, 1}(x), u_{n, 2}(x), \ldots,
$$

i.e. $\left(u_{n, j}, v_{m, s}\right)=\delta_{n, m} . \delta_{j, s}, n, m=1,2, \ldots, j, s=1,2$, where $(\cdot, \cdot)$ denotes the inner product in the space $L_{2}(0,1)$ and $\delta_{n, m}$ is the Kronecker symbol. It is well known, see [14, p. 84] or [27, p. 99], that (89) is the system of root functions of the differential operator $L^{*}$, which is the adjoint operator to $L$. The differential operator $L^{*}$ is generated by the differential expression

$$
l^{*}(z)=z^{\mathrm{iv}}+\left(\overline{p_{2}(x)} z\right)^{\prime \prime}-\left(\overline{p_{1}(x)} z\right)^{\prime}+\overline{p_{0}(x)} z
$$

and the adjoint boundary conditions

$$
\begin{gathered}
U_{0}^{*}(z) \equiv z(1)-(-1)^{\sigma} z(0)=0, \quad U_{1}^{*}(z) \equiv z^{\prime}(1)-(-1)^{\sigma} z^{\prime}(0)+\overline{\alpha_{3,2}} z(0)=0, \\
U_{2}^{*}(z) \equiv z^{\prime \prime}(1)-(-1)^{\sigma} z^{\prime \prime}(0)+\overline{\alpha_{2,1}} z^{\prime}(0)+\beta_{2,0} z(0)=0, \\
U_{3}^{*}(z) \equiv z^{\prime \prime \prime}(1)-(-1)^{\sigma} z^{\prime \prime \prime}(0)+\overline{\alpha_{1,0}} z^{\prime \prime}(0)+\beta_{3,1} z^{\prime}(0)+\beta_{3,0} z(0)=0,
\end{gathered}
$$


where $\beta_{2,0}, \beta_{3,1}$ and $\beta_{3,0}$ are some numbers which depend only on the coefficients of the differential operator (2)-(3). Thus, for $j=1,2$ and for sufficiently large numbers $n$, the following relations are valid: $l^{*}\left(v_{n, j}\right)=\overline{\lambda_{n, j}} v_{n, j}, U_{s}^{*}\left(v_{n, j}\right)=0$. From the form of the differential operator $L^{*}$ and Theorem 1.1, it follows that for sufficiently large numbers $n$ the equalities

$$
\overline{v_{n_{1}+n, 1}(x)}=r_{n_{1}+n, 1}\left(\sin (2 n-\sigma) \pi x+O\left(n^{-1}\right)\right), \quad \overline{v_{n_{2}+n, 2}(x)}=r_{n_{1}+n, 2}\left(\cos (2 n-\sigma) \pi x+O\left(n^{-1}\right)\right)
$$

hold, where $r_{n_{j}+n, j}, j=1,2$, are some numbers determined by the equality $\left(u_{n_{j}+n, j}, v_{n_{j}+n, j}\right)=1, j=1,2$. From here and the asymptotic formulae (88) and (91), for sufficiently large $n, r_{n_{j}+n, j}=\sqrt{2}+O\left(n^{-1}\right), j=1,2$. Consequently, from (91), again for sufficiently large $n$,

$$
\overline{v_{n_{1}+n, 1}(x)}=\sqrt{2} \sin (2 n-\sigma) \pi x+O\left(n^{-1}\right), \quad \overline{v_{n_{2}+n, 2}(x)}=\sqrt{2} \cos (2 n-\sigma) \pi x+O\left(n^{-1}\right) .
$$

Let

$$
\begin{gathered}
g_{0}(x)=1, \quad g_{2 n-1}(x)=\sqrt{2} \sin 2 n \pi x, \quad g_{2 n}(x)=\sqrt{2} \cos 2 n \pi x, \quad n=1,2, \ldots, \\
\tilde{g}_{2 n-1}=\sqrt{2} \sin (2 n-1) \pi x, \quad \tilde{g}_{2 n}=\sqrt{2} \cos (2 n-1) \pi x, \quad n=1,2, \ldots
\end{gathered}
$$

Each of the systems (93) and (94) is an orthonormal basis of the space $L_{2}(0,1)$. From the asymptotic formulae (88), (92), it is obvious that each of the systems (89) and (90) satisfies the Bessel inequality. Namely, for an arbitrary function $f(x) \in L_{2}(0,1)$,

$$
\sum_{n=1}^{\infty} \sum_{j=1}^{2}\left|\left(f, u_{n, j}\right)\right|^{2}<\infty, \quad \sum_{n=1}^{\infty} \sum_{j=1}^{2}\left|\left(f, v_{n, j}\right)\right|^{2}<\infty .
$$

Furthermore, each of the systems (89) and (90) is complete in the space $L_{2}(0,1)$, see e.g. [28]. Consequently [9, Chapter $\mathrm{VI}$, $\S 2.2$, Theorem 2.1] each of these systems forms a Riesz basis of the space $L_{2}(0,1)$.

Let us prove Corollary 1.3. Consider the case $\sigma=0$. The case $\sigma=1$ can be checked in the same way. Assume that $n_{1} \geq 0$ and $n_{2} \geq 0$. From the asymptotic formulae (88), and the definition of $\left\{g_{k}(x)\right\}_{k=0}^{\infty}$, see (93), we obtain

$$
\sum_{n=1}^{\infty}\left(\left\|u_{n_{1}+n, 1}-g_{2 n-1}\right\|^{2}+\left\|u_{n_{2}+n, 2}-g_{2 n}\right\|^{2}\right) \leq \text { const } \sum_{n=1}^{\infty} \frac{1}{n^{2}}<+\infty .
$$

It is easy to see that $n_{1}+n_{2}$ root functions of the operator $L$ and one function from system (93) are absent in (95). Let $n_{1}+n_{2}>1$. In this case, by (95), the system $S$ generated by all functions except $n_{1}+n_{2}-1$ functions from the system (90) is quadratically close to the system (93). Since (93) is an orthonormal basis of $L_{2}(0,1)$, then $S$ also forms a Riesz basis of $L_{2}(0,1)\left[9\right.$, Chapter $\mathrm{VI}, \S 2.4$, Theorem 2.3]. The latter contradicts the basicity of the system $(90)$ in $L_{2}(0,1)$.

Let $n_{1}=n_{2}=0$. Since (90) is a Riesz basis of the space $L_{2}(0,1)$, then again by (95), the system $\left\{g_{k}(x)\right\}_{k=1}^{\infty}$ is a basis and this contradicts the basicity of the system $\left\{g_{k}(x)\right\}_{k=0}^{\infty}$ in $L_{2}(0,1)$. All the remaining cases can be investigated in a similar way.

Thus, it holds $n_{1}+n_{2}=1$. Therefore, without loss of generality we can assume that $n_{1}=0$ and $n_{2}=1$. Consequently, from (88) we have

$$
u_{n, 1}(x)=\sqrt{2} \sin (2 n-\sigma) \pi x+O\left(n^{-1}\right), \quad u_{n+1-\sigma, 2}(x)=\sqrt{2} \cos (2 n-\sigma) \pi x+O\left(n^{-1}\right) .
$$

Similarly, the asymptotic formulae (92) will take the forms

$$
\overline{v_{n, 1}(x)}=\sqrt{2} \sin (2 n-\sigma) \pi x+O\left(n^{-1}\right), \quad \overline{v_{n+1-\sigma, 2}(x)}=\sqrt{2} \cos (2 n-\sigma) \pi x+O\left(n^{-1}\right) .
$$

Next we prove that the system of root functions of the differential operator $L$ forms a basis of the space $L_{p}(0,1)$, where $1<p<\infty$ and $p \neq 2$. As above, we consider only the case $\sigma=0$. The case $\sigma=1$ is similar. 
Note that $(93)$ is a basis of the space $L_{p}(0,1)$ for any $p \in(1, \infty)[1$, Chapter VIII, $\S 20$, Theorem 2]. Consequently $\left[12\right.$, Chapter I, $\S 4$, Theorem 6] there exists a constant $M_{p}>0$ ensuring the inequality

$$
\left\|\sum_{n=0}^{N}\left(f, g_{n}\right) g_{n}\right\|_{p} \leq M_{p}\|f\|_{p}, \quad N=1,2, \ldots
$$

for any function $f(x) \in L_{p}(0,1)$, where $\|\cdot\|_{p}$ means the norm in $L_{p}(0,1)$. We now fix $p \in(1,2)$. Since the system (90) is complete in the space $L_{2}(0,1)$, then this system is complete in $L_{p}(0,1)$ as well. Moreover, it is easy to see that

$$
\left\|\left(f, v_{n, j}\right) u_{n, j}\right\|_{p} \leq \mathrm{const}\|f\|_{p}
$$

where $n=1,2, \ldots$ and $j=1,2$. Consequently [12, Chapter VIII, $\S 4$, Theorem 6], in order to prove the basicity of this system in $L_{p}(0,1)$, it is enough to prove the existence of a constant $M>0$ ensuring the inequality

$$
\left\|\sum_{n=1}^{m} \sum_{j=1}^{2}\left(f, v_{n, j}\right) u_{n, j}\right\|_{p} \leq M\|f\|_{p}, \quad m=1,2, \ldots,
$$

for $f(x) \in L_{p}(0,1)$. Note that instead of this inequality, under the same conditions, it is enough to prove the inequality

$$
J_{m}(f)=\left\|\sum_{n=1}^{m}\left\{\left(f, v_{n, 1}\right) u_{n, 1}+\left(f, v_{n+1,2}\right) u_{n+1,2}\right\}\right\|_{p} \leq M^{\prime}\|f\|_{p},
$$

where $m=1,2, \ldots$ and $M^{\prime}$ is a certain positive constant. From (96)-(97) and (93) we have

$$
\begin{array}{ll}
u_{n, 1}(x)=g_{2 n-1}(x)+O\left(n^{-1}\right), & u_{n+1,2}=g_{2 n}(x)+O\left(n^{-1}\right), \\
\overline{v_{n, 1}(x)}=g_{2 n-1}(x)+O\left(n^{-1}\right), & \overline{v_{n+1,2}(x)}=g_{2 n}(x)+O\left(n^{-1}\right) .
\end{array}
$$

Consequently,

$$
J_{m}(f) \leq J_{m, 1}(f)+J_{m, 2}(f)+J_{m, 3}(f)+J_{m, 4}(f),
$$

where $m=1,2, \ldots$ and

$$
\begin{array}{cl}
J_{m, 1}(f)=\left\|\sum_{n=1}^{2 m}\left(f, g_{n}\right) g_{n}\right\|_{p}, & J_{m, 2}(f)=\left\|\sum_{n=1}^{2 m}\left(f, g_{n}\right) O\left(n^{-1}\right)\right\|_{p}, \\
J_{m, 3}(f)=\left\|\sum_{n=1}^{2 m}\left(f, O\left(n^{-1}\right)\right) g_{n}\right\|_{p}, \quad J_{m, 4}(f)=\left\|\sum_{n=1}^{2 m}\left(f, O\left(n^{-1}\right)\right) O\left(n^{-1}\right)\right\|_{p} .
\end{array}
$$

By (98),

$$
J_{m, 1}(f) \leq \text { const }\|f\|_{p}
$$

From the Riesz theorem [33, Chapter XII, §2, Theorem 2.8] it follows that

$$
J_{m, 2}(f) \leq \mathrm{const} \sum_{n=1}^{2 m}\left|\left(f, g_{n}\right)\right| n^{-1} \leq \mathrm{const}\left(\sum_{n=1}^{2 m}\left|\left(f, g_{n}\right)\right|^{q}\right)^{1 / q}\left(\sum_{n=1}^{2 m} n^{-p}\right)^{1 / p} \leq \mathrm{const}\|f\|_{p},
$$

where $1 / p+1 / q=1$. Further,

$$
J_{m, 3}(f) \leq\left\|\sum_{n=1}^{2 m}\left(f, O\left(n^{-1}\right)\right) g_{n}\right\|_{2}=\left(\sum_{n=1}^{2 m}\left|\left(f, O\left(n^{-1}\right)\right)\right|^{2}\right)^{1 / 2} \leq \text { const }\|f\|_{1}\left(\sum_{n=1}^{2 m} n^{-2}\right)^{1 / 2} \leq \text { const }\|f\|_{p} .
$$


Moreover,

$$
J_{m, 4} \leq \text { const }\|f\|_{1} \sum_{n=1}^{2 m} n^{-2} \leq \text { const }\|f\|_{p} .
$$

The inequality (99) is a consequence of the inequalities (100)-(104). Thus, the basicity of the system (90) in the space $L_{p}(0,1)$ for $1<p<2$ is proved.

Let $2<p<\infty$ and $1 / p+1 / q=1$. Note that $1<q<2$ and (89) is the system of root functions of the differential operator $L^{*}$. As it has been proved above, the system of root functions of such operator forms a basis of the space $L_{r}(0,1)$ for any $r \in(1,2)$, in particular $r=q$. Thus, (89) is a basis of $L_{q}(0,1)$. Consequently, the system $(90)$ which is biorthogonally conjugate to (89) forms a basis of $L_{p}(0,1)$.

\section{References}

[1] Bari N.K., A Treatise on Trigonometric Series. Vol. II, Macmillan, New York, 1964

[2] Dernek N., Veliev O.A., On the Riesz basisness of the root functions of the nonself-adjoint Sturm-Liouville operator, Israel J. Math., 2005, 145, 113-123

[3] Djakov P., Mityagin B.S., Instability zones of one-dimensional periodic Shrödinger and Dirac operators, Russian Math. Surveys, 2006, 61(4), 663-766

[4] Djakov P., Mityagin B., Convergence of spectral decompositions of Hill operators with trigonometric polynomials as potentials, Doklady Math., 2011, 83(1), 5-7

[5] Djakov P., Mityagin B., Convergence of spectral decompositions of Hill operators with trigonometric polynomial potentials, Math. Ann., 2011, 351(3), 509-540

[6] Djakov P., Mityagin B., Criteria for existance of Riesz bases consisting of root functions of Hill and 1D Dirac operators, preprint available at http://arxiv.org/abs/1106.5774

[7] Dunford N., Schwartz J.T., Linear Operators. Part III, Wiley Classics Lib., John Wiley \& Sons, New York, 1988

[8] Gesztesy F., Tkachenko V., A Schauder and Riesz basis criterion for non-self-adjoint Schrödinger operators with periodic and antiperiodic boundary conditions, preprint available at http://arxiv.org/abs/1104.4846

[9] Gohberg I.C., Kreŭn M.G., Introduction to the Theory of Linear Nonselfadjoint Operators, Transl. Math. Monogr., 18, American Mathematical Society, Providence, 1969

[10] Il'in V.A., Kritskov L.V., Properties of spectral expansions corresponding to non-self-adjoint differential operators, J. Math. Sci. (N.Y.), 2003, 116(5), 3489-3550

[11] Ionkin N.I., The solution of a certain boundary value problem of the theory of heat conduction with a nonclassical boundary condition, Differ. Uravn., 1977, 13(2), 294-304 (in Russian)

[12] Kashin B.S., Saakyan A.A., Orthogonal Series, Transl. Math. Monogr., 75, American Mathematical Society, Providence, 1989

[13] Kerimov N.B., Mamedov Kh.R., On the Riesz basis property of the root functions in certain regular boundary value problems, Math. Notes, 1998, 64(4), 483-487

[14] Keselman G.M., On the unconditional convergence of eigenfunction expansions of certain differential operators, Izv. Vyssh. Uchebn. Zaved. Mat., 1964, 39(2), 82-93 (in Russian)

[15] Kiraç A.A., Riesz basis property of the root functions of non-selfadjoint operators with regular boundary conditions, Int. J. Math. Anal. (Ruse), 2009, 3(21-24), 1101-1109

[16] Makin A.S., On a class of boundary value problems for the Sturm-Liouville operator, Differ. Uravn., 1999, 35(8), 1058-1066 (in Russian)

[17] Makin A.S., On spectral decompositions corresponding to non-self-adjoint Sturm-Liouville operators, Dokl. Math., 2006, 73(1), 15-18

[18] Makin A.S., Convergence of expansions in the root functions of periodic boundary value problems, Dokl. Math., 2006, 73(1), 71-76

[19] Makin A.S., On the basis property of systems of root functions of regular boundary value problems for the SturmLiouville operator, Differ. Equ., 2006, 42(12) 1717-1728 
[20] Makin A.S., Characterization of the spectrum of regular boundary value problems for the Sturm-Liouville operator, Differ. Equ., 2008, 44(3), 341-348

[21] Makin A.S., Asymptotics of the spectrum of the Sturm-Liouville operator with regular boundary conditions, Differ. Equ., 2008, 44(5), 645-658

[22] Mamedov Kh.R., On the basis property in $L p(0,1)$ of the root functions of a class non self adjoint Sturm-Liouville operators, Eur. J. Pure Appl. Math., 2010, 3(5), 831-838

[23] Mamedov Kh.R., Menken H., On the basisness in $L_{2}(0,1)$ of the root functions in not strongly regular boundary value problems, Eur. J. Pure Appl. Math., 2008, 1(2), 51-60

[24] Menken H., Accurate asymptotic formulas for eigenvalues and eigenfunctions of a boundary-value problem of fourth order, Bound. Value Probl., 2010, \# 720235

[25] Menken H., Mamedov Kh.R., Basis property in $L_{p}(0,1)$ of the root functions corresponding to a boundary-value problem, J. Appl. Funct. Anal., 2010, 5(4), 351-356

[26] Mikhailov V.P., On Riesz bases in $\mathcal{L}_{2}(0,1)$, Dokl. Akad. Nauk SSSR, 1962, 144(5), 981-984 (in Russian)

[27] Naimark M.A., Linear Differential Operators, 2nd ed., Nauka, Moskow, 1969 (in Russian)

[28] Shkalikov A.A., Basis property of eigenfunctions of ordinary differential operators with integral boundary conditions, Vestnik Moskov. Univ. Ser. I Mat. Mekh., 1982, 6, 12-21

[29] Shkalikov A.A., Veliev O.A., On the Riesz basis property of eigen- and associated functions of periodic and antiperiodic Sturm-Liouville problems, Math. Notes, 2009, 85(5-6), 647-660

[30] Veliev O.A., On the nonself-adjoint ordinary differential operators with periodic boundary conditions, Israel J. Math., 2010, 176, 195-207

[31] Veliev O.A., Asymptotic analysis of non-self-adjoint Hill operators, preprint available at http://arxiv.org/abs/ 1107.2552

[32] Veliev O.A., Duman M.T., The spectral expansion for a nonself-adjoint Hill operator with a locally integrable potential, J. Math. Anal. Appl., 2002, 265(1), 76-90

[33] Zygmund A., Trigonometric Series. II, 2nd ed., Cambridge University Press, New York, 1959 